\title{
Poling Quality Evaluation of Periodically Poled Lithium Niobate Using Diffraction Method
}

\author{
Krishnamoorthy Pandiyan, Yeon-Suk Kang, Hwan-Hong Lim, Byeong-Joo Kim, Om Prakash, \\ and Myoungsik Cha* \\ Department of Physics, Pusan National University, Busan 609-735, Korea.
}

(Received August 13, 2008 : revised August 13, 2008 : accepted September 8, 2008)

\begin{abstract}
We demonstrated a simple way of evaluating the duty cycle error in periodically polled lithium niobate (PPLN) based on the method of binary phase diffraction grating. To demonstrate this method, $-\mathrm{Z}$ face etched PPLN of desired periods were fabricated by the standard electric field poling technique. The etched PPLN was considered as a surface-relief binary phase grating. The diffraction patterns were recorded for different spatial locations along the length of the sample. The experimentally observed efficiencies of the diffracted orders were compared with the theoretically calculated values to estimate the duty cycle error.
\end{abstract}

Keywords: Periodically poled lithium niobate; Diffraction grating

OCIS codes : (050.1950) Diffraction gratings ; (130.3730) Lithium niobate ; (160.4330) Nonlinear optical materials

\section{INTRODUCTION}

The development of domain-engineered crystals has gained significant importance not only in nonlinear optical frequency conversion processes [1], but also in linear polarization control or modulation devices $[2,3]$. Quasi phase matching (QPM) devices have been successfully realized in ferroelectric crystals such as lithium niobate $\left(\mathrm{LiNbO}_{3}\right)$, lithium tantalate $\left(\mathrm{LiTaO}_{3}\right)$ and potassium titanyl phosphate $\left(\mathrm{KTiOPO}_{4}\right)$ by the process of electric field poling, in which the domain structures are designed to compensate the mismatch among the wave vectors of the interacting light beams. Utilizing this approach, QPM grating periods with a desired duty cycle can be fabricated. In order to achieve maximum conversion efficiency, the periodically poled structure must have a uniform grating period and duty cycle throughout the poled region. Therefore, it is essential to check the quality of the poled QPM device. There are several methods to visualize the resulting domain structures such as second harmonic generation microscopy [4], confocal luminescence microscopy [5], optical near field microscopy [6] etc., However in these techniques only a small area of the poled pattern can be visualized.

*Corresponding author: mcha@pusan.ac.kr
An alternative approach to investigate the poled QPM device in terms of refractive index step created by applying an electric field have been described by Müller et al [7].

In QPM devices two kinds of error (Fig. 1) are inherent, one is period error and the other is duty cycle error. In general a PPLN fabricated by the electric field poling method is relatively immune to period error because the periodicity is precisely determined by the lithographically defined grating electrode, while duty cycle error is predominant. The effects of duty cycle error on parametric gain have been analyzed by Fejer et al [1], the overall gain reduction factor $\eta_{d}$ due to duty cycle error is,

$$
\eta_{d}=\left[\sin ^{2}(\pi n D)\right]\left[1-\left(\frac{\pi^{2}}{2}\right)\left(\frac{\sigma_{l}}{l_{c}}\right)\right]
$$

where $D$ is the average duty cycle, $m$ is the order of $\mathrm{QPM}, \sigma_{l}$ is the variance in the domain width, and $l_{c}$ is the coherence length. Therefore it is very important to estimate the error from the (1:1) duty cycle to get the maximum conversion efficiency. In this communication, we report a simple technique to quantify the duty cycle error by performing a far-field diffraction experiment and analyzing it with the model of the binary phase grating. 


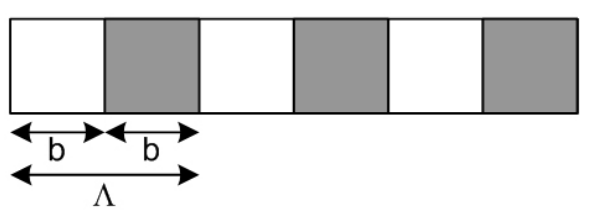

(a)

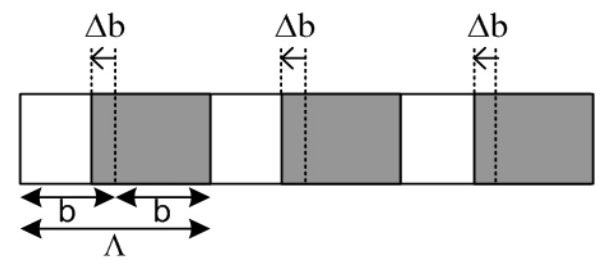

(b)

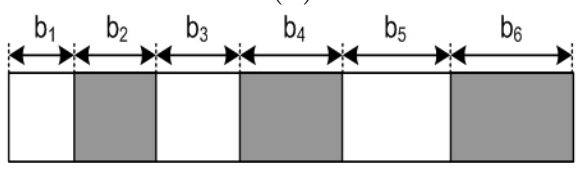

(c)

FIG. 1. Schematic diagram of the QPM device (a) Ideal structure, (d) Duty cycle error and (c) Period error.

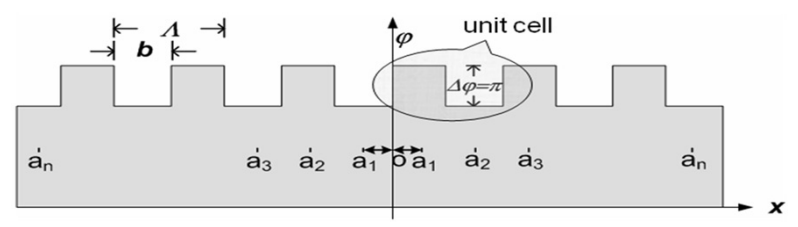

FIG. 2. A binary phase grating model for a etched PPLN.

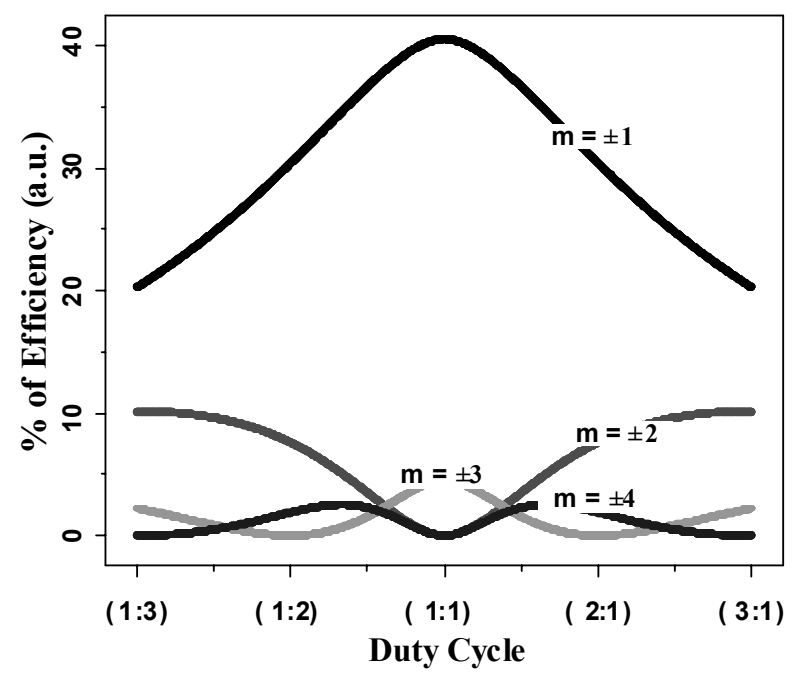

FIG. 3. Percentage of diffraction efficiency versus change in duty cycle (theory).

\section{THEORY}

To get an idea about the diffraction pattern a theoretical model of the binary phase grating (Fig. 2) was used. The far-field diffraction amplitude of the unit cell of the binary phase grating is given by $[8,9]$,

$$
\begin{aligned}
& E(\theta) \propto \frac{b}{\Lambda} \operatorname{sinc}\left(\frac{\pi b \theta}{\lambda}\right) \exp \left(i a_{1} k b \theta\right)-\left(\frac{\Lambda-b}{\Lambda}\right) \\
& \operatorname{sinc}\left[\frac{\pi(\Lambda-b) \theta}{\lambda}\right] \exp \left[i a_{1} k(\Lambda-b) \theta\right.
\end{aligned}
$$

Where,

$\Lambda$ is the period of the grating,

$b$ is the width of the etched domain,

$\varphi=2 \pi d(n-1) / \lambda$ is the phase depth,

$d$ is the etch depth,

$n$ is the index of the material,

$a_{i}$ is the position of the domain from the origin "O",

$\theta$ is the angle of diffraction,

$k=2 \pi / \lambda$ is the wave vector.

Fig. 3 shows the general behavior of diffraction efficiency variation corresponding to duty cycle fluctuation; where the efficiency variation between the orders depicts the symmetric nature of the duty cycle variation. From the graph we noticed that the spectrum of (1:1) duty cycle gives only odd order peaks while even orders are zero. Both the theoretical and experimental analyses shows that the diffracted pattern for the duty cycles such as $(1: 2) \&(2: 1)$ have the same kind of efficiency distribution between the orders [10]. It is also obvious that the variation in duty cycle directly affects the diffraction efficiency between the orders. These variations in efficiency between the orders were taken into account to predict the duty cycle error. For simplicity we have neglected the zeroth order spectral response.

\section{EXPERIMENT AND DISCUSSION}

Z-cut wafers of congruent lithium niobate crystal (Yamaju Ceramics) were used for periodic poling. The electrode for domain reversal was made by photolithography process. The wafer was well cleaned and a photoresist (Clariant, AZ $5214 \mathrm{E}$ ) was deposited on $+\mathrm{Z}$ face of the wafer. A DC electric field was applied to the sample using saturated lithium chloride as a liquid electrode. The complete experimental setup of our poling system has been given Fig. 4. Before fixing the wafer inside the fixture the area to be poled, A, was calculated. The spontaneous polarization of our poling system was estimated to be, $P_{s}=78 \mu \mathrm{C} / \mathrm{cm}^{2}[11]$.

In electric field poling, domain reversal occurs only when the applied field exceeds a certain value referred to as the coercive field, which is $\sim 21 \mathrm{kV} / \mathrm{mm}$ for $\mathrm{LiNbO}_{3}$ at room temperature. In order to apply the proper amount of charge $Q$ to get (1:1) duty cycle PPLN device, a poling current $I$ must be applied for a particular time 


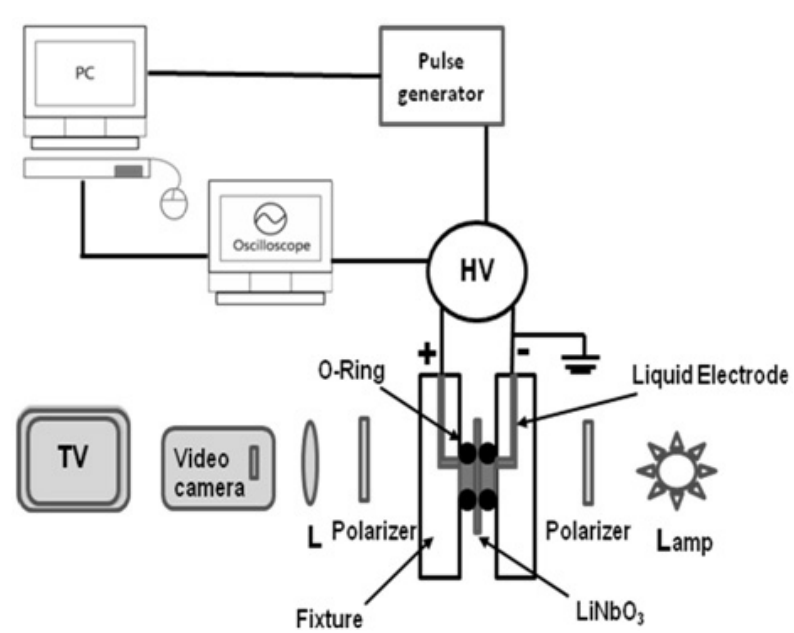

FIG. 4. Schematic diagram of the electric-field poling setup.
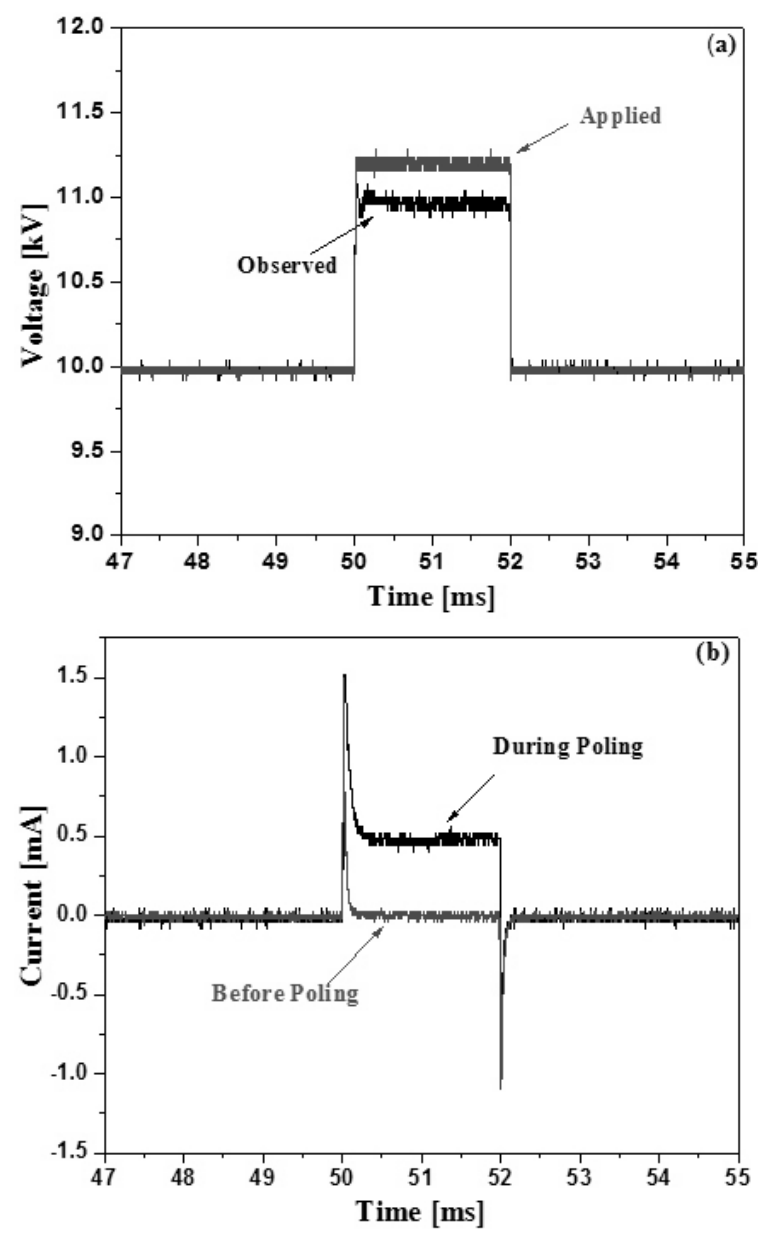

FIG. 5. Pulse structure for the poling of $0.5 \mathrm{~mm}$ thick $\mathrm{LiNbO}_{3}$ : (a) applied and observed voltage during poling, (b) observed current before and during poling.

$T$ such that $I T=Q=2 A P_{s}$. In general, the specific poling current, $I$, depends on the applied voltage, the coercive field and the sample thickness. As a result, poling

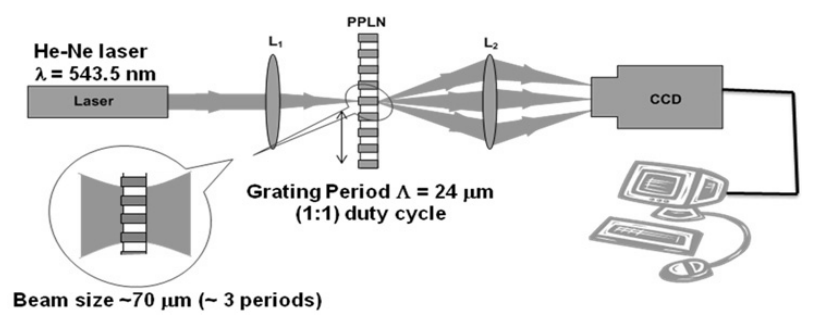

Fig. 6. A schematic diagram of experimental setup used for the duty cycle error measurement.

TABLE 1. Theoretically calculated diffraction efficiencies of the orders 1, 2 and 3 for different duty cycles.

\begin{tabular}{l|c|c|c}
\hline $\begin{array}{l}\text { Efficiency } \\
\text { Duty } \\
\text { cycle }\end{array}$ & $\begin{array}{c}1^{\text {st }} \text { order } \\
\text { (a.u.) }\end{array}$ & $\begin{array}{c}2^{\text {nd }} \text { order } \\
(\text { a.u. })\end{array}$ & $3^{\text {rd }}$ order (a.u.) \\
\hline$(1: 1)$ & 1 & 0 & 0.11 \\
\hline$(1: 2)$ or $(2: 1)$ & 0.75 & 0.19 & 0 \\
\hline$(1: 3)$ or $(3: 1)$ & 0.5 & 0.25 & 0.05 \\
\hline
\end{tabular}

The sample with a single voltage pulse for a specified time almost gives a total poling charge different from the desired charge $Q$. So applying several voltage pulses, using the measured poling current from previous pulses to determine the duration of the last few pulses will give the desired poling pattern [12]. Fig. 5 shows a typical pulse structure of voltage and current. The increase in current or decrease in voltage indicates the progress in domain reversal process [13].

Even though we do the poling process carefully, one can observe the fluctuation in the poled pattern. To explore the fluctuation in the PPLN, the $-\mathrm{Z}$ surface was etched with hydro-fluoric solution. It has been reported that a $48 \%$ of pure hydro-fluoric solution is the best known etchant for lithium niobate. It etches smoothly at $-\mathrm{Z}$ face of PPLN whereas the inverted domain etching rate is negligible [14]. The etched PPLN can be considered as a surface-relief binary phase grating.

Fig. 6 shows the experimental setup of the far field diffraction measurement. A He-Ne laser (Melles Griot, $\lambda=543.5 \mathrm{~nm}$ ) beam of focused spot size of $\sim 70 \mu \mathrm{m}$ was used to illuminate the PPLN in order to get the diffraction pattern. The diffracted beams were focused by a lens on a CCD camera which was interfaced to a personal computer. The diffraction patterns were recorded for different spatial locations, namely fifty locations along the length of the sample.

The resolving power and position of the different order spectra of the binary phase grating with different duty cycle can be explained by the phasor analysis using a Fourier transform [15]. In the present study, the PPLN with the period of $24.0 \mu \mathrm{m}$ of duty cycle (1:1) has been used. In table 1 the efficiencies are calculated by normalizing the theoretical spectra corresponding to the first 


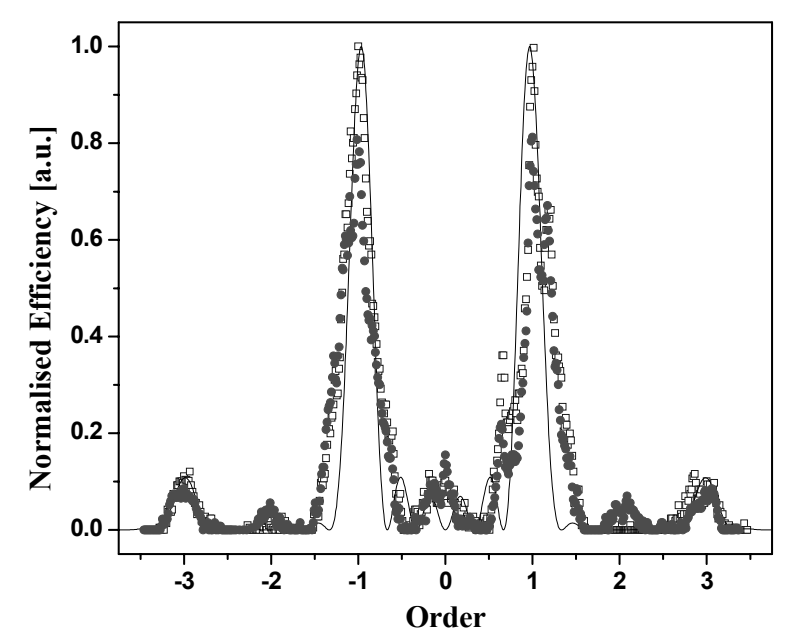

FIG. 7. Diffraction spectrum for theoretically calculated (solid line), reference spectrum (open square) and spectrum with duty cycle error (solid circle).

order efficiency of (1:1) duty cycle as it has given maximum efficiency. For an ideal (1:1) structure the normalized efficiency of the first, the second and the third orders were found to be 1,0 , and 0.11 respectively. For the grating period of $24.0 \mu \mathrm{m}$ with the (1:1) duty cycle, the domain width of $12.0 \mu \mathrm{m}$ for each domain that means (12.0:12.0) for both the reversed and the unreversed domain. In the case of (1:2) or (2:1) the width of the domains can be either (8.0:16.0) or (16.0:8.0) which corresponds to an error of $\Delta b=4.0 \mu \mathrm{m}$ in each domain or $8 \mu \mathrm{m}$ error in the duty cycle, which represents $33.33 \%$ of error from the ideal (1:1) structure. Similarly, a duty cycle of (1:3) or (3:1) represents $50 \%$ of error from the ideal structure.

Similarly, in each experimentally observed spectrum the efficiencies between the orders were compared; the one which exactly satisfied the theoretical values of (1:1) duty cycle was taken as a reference. The other spectra were normalized with respect to this reference. Then the efficiency variations between the orders were compared with the reference spectrum. In our observation most of the spectra were matched with the reference spectrum, whereas few spectra did not match with the ideal, which reflects the amount of duty cycle fluctuation in the PPLN. The overall variation from all the spectra gives the average error in duty cycle or standard deviation. Typical comparison data is given in Fig. 7, the data points contain the information about the duty cycle error of a particular location, where the theoretical curve has been drawn for three unit cells. The first order decreased from 1 to 0.812 which represents a $4.0 \mu \mathrm{m}$ duty cycle error from the ideal structure i.e. (10.0:14.0) or (14.0:10.0) for the period of $24.0 \mu \mathrm{m}$. Similarly we compared all the spectra with the ideal structure, based on comparison we could observe average duty cycle error of $\Delta b \sim 1.44 \mu \mathrm{m}$, corresponding to a $12 \%$ error for a QPM period of $24.0 \mu \mathrm{m}$.
The main reasons for duty cycle error in the QPM devices are; the substrate inhomogeneity and the photolithography process. Substrate inhomogeneity causes lack of nucleation site density and uneven movement of domain walls, this leads to large fluctuation in the duty cycle [16]. The uneven movement of domain walls can be seen in real time in-situ monitoring system using a proper combination of polarizer and analyzer. Further the in-homogeneity causes improper tip propagation and it wanders from a strictly z-directed domain path. Further investigations are necessary to understand this effect. In photolithography the processing steps, including soft baking, UV exposure, developing and hard baking also cause this error.

The accuracy of the duty cycle evaluation in this experiment mainly depends on the aberration of the lens, sensitivity of the CCD camera, and the averaging effect within the beam size. Aberration from the lens plays the most important role, because our estimation purely depends on the intensity ratio between the orders. Since aberration can alter the intensity of each diffracted order, this effect could be largely reduced by using a longer focal length lens. Measuring the peaks with CCD camera is similar to using a power meter for measurement. Thus one can expect an error less than one percent from the CCD camera when it works in the linear regime. The averaging effect exists, but is considered to be small if we assume that there is no abrupt pattern change within the beam diameter. (Of course, we are not picking up all the details of each cell.) Overall, we estimate that the accuracy of our estimation would be $\pm 1 \%$ at worst case. (Laser fluctuation does not matter, because we are measuring the ratio of the intensities of the orders.)

So far, we have investigated duty cycle error with the binary phase grating diffraction model by launching a beam of small diameter to the PPLN. In the future, the same method will be used with a larger diameter beam covering the whole length of the QPM device to investigate the average duty cycle error in a single spectrum. This will be used to measure the change in efficiency and bandwidth of the frequency conversion process. These changes are the direct consequences of various duty cycle errors in QPM devices.

\section{CONCLUSION}

PPLN of desired periods and duty cycles were fabricated by applying the proper amount of current to the electric field poling system. A simple method was proposed to check the quality of the PPLN by recording the far field diffraction pattern of the etched PPLN and treating it as a surface-relief binary phase grating. Utilizing this method, the average duty cycle error of $12 \%$ was observed for a PPLN device of the period $24.0 \mu \mathrm{m}$. In principle, this technique could be used as a quality 
inspection tool for the periodically and aperiodically poled ferroelectric QPM devices.

\section{ACKNOWLEDGMENT}

This work was supported by Korean Research Foundation Grant No. KRF-2006-005-J02803, and by the Ministry of Commerce, Industry and Energy of Korea through the Industrial Technology Infrastructure Building Program.

\section{REFERENCES}

[1] M. M. Fejer, G. A. Magel, D. H. Jundt, and R. L. Byer, "Quasi-phase-matched second harmonic generation: tuning and tolerances," IEEE J. Quantum. Electron., vol. 28, no. 11, pp. 2631-2654, 1992.

[2] C.-S. Kee, J. Lee , and Y. L. Lee, "Characteristics of Sŏlc filters in $\mathrm{X}^{(2)}$ nonlinear photonic crystals," J. Opt. Soc. Korea, vol. 11, no 3, pp. 130-132, 2007.

[3] I. M. Jung and D.-S. Shin, "Efficient optical intensity modulator based on the electrically tunable $\mathrm{LiNbO}_{3}$ reflection grating for analog fiber-optic links," J. Opt. Soc. Korea, vol. 11, no. 1, pp. 1-5, 2007.

[4] A. Rosenfeldt and M. Florsheimer, "Nondestructive remote imaging of ferroelectric domain distributions with high three-dimensional resolution," Appl. Phys. B., vol. 73, no. 5-6, pp. 523-529, 2001.

[5] V. Dierolf and C. Sandmann, "Inspection of periodically poled waveguide devices by confocal luminescence microscopy," Appl. Phys., B., vol. 78, no. 3-4, pp. 363-366, 2004 .
[6] T. J. Yang, V. Gopalan, P. J. Swart, and U. Mohideen, "Direct Observation of Pinning and Bowing of a Single Ferroelectric Domain Wall," Phys. Rev. Lett., vol. 82, no. 20, pp. 4106-4109, 1999.

[7] M. Müller, E. Soergel, K. Buse, C. Langrock, and M. M. Fejer, "Investigation of periodically poled lithium niobate crystals by light diffraction," J. Appl. Phys., vol. 97, no. 4, pp. 044102, 2005.

[8] J. W. Goodman, "Introduction to Fourier Optics," $2^{\text {nd }}$ ed.,( McGraw-Hill, Singapore, 1996)

[9] D. C. Oshea, T. J. Suleski, A. D. Kathman, and D. W. Prathes, "Diffractive Optics: Design, Fabrication and Test," SPIE Publication, 2003.

[10] M .J. Jin, O. Y. Jeon, B. J. Kim, and M. Cha, "Fabrication of Periodically Poled Lithium Niobate Crystal and Poling-Quality Evaluation by Diffraction Measurement," J. Korean Phys. Soc., vol. 47, pp. S336-S339, 2005.

[11] J. H. Ro, Ph.D. thesis, Pusan National University, 2001.

[12] M. J. Missey, S. Russell, V. Dominic, R. G. Batchko, and K. L. Schepler, "Real-time visualization of domain formation in periodically poled lithium niobate," Opt. Express., vol. 6, no. 10, pp. 186-195, 2000.

[13] V. Gopalan, Q. X. Jia, and T. E. Mitchell, "In situ video observation of $180^{\circ}$ domain kinetics in congruent $\mathrm{LiNbO}_{3}$ crystals," Appl. Phys. Lett., vol. 75, no. 16, pp. 2482-2484, 1999.

[14] C. L. Sones, S. Mailis, W. S. Brocklesby, R. W. Eason, and J. R. Owe, "Differential etch rates in z-cut $\mathrm{LiNbO}_{3}$ for variable $\mathrm{HF} / \mathrm{HNO}_{3}$ concentrations," J. Material Chem., vol. 12, pp. 295-298, 2002.

[15] M. M. Sanchezlopez, J. Cos, J. A. Davis, D. A. Miller, and I. Moreno, "Fourier analysis of harmonic frequency transmission dielectric structures," Appl. Opt., vol. 44, no. 18, pp. 3774-3783, 2005.

[16] G. D. Miller, Ph.D. thesis, Stanford University, 1998. 